\title{
Editorial
}

\section{Excess Body Weight and Gastrointestinal Disease}

\author{
Christoph-Thomas Germer ${ }^{a}$ Hans Scherübl ${ }^{b}$ \\ aKlinik für Allgemein-, Viszeral-, Transplantations-, Gefäß- und Kinderchirurgie, Universitätsklinikum Würzburg, \\ Würzburg, Germany; ${ }^{b}$ Klinik für Innere Medizin II, Gastroenterologie, Gl Onkologie, Diabetologie und Infektiologie, \\ Vivantes Klinikum Am Urban, Akademisches Lehrkrankenhaus der Charité, Berlin, Germany
}

The global number of obese adults increased about 6 -fold over the last 4 decades. Nowadays many people enjoy an easy access to high-calorie foods and often indulge in a physically inactive, sedentary lifestyle. This has led to an increased number of deaths from cardiovascular disease, diabetes, the metabolic syndrome, and cancer. It is becoming increasingly evident that excess body weight (EBW) also increases the risk of various debilitating gastrointestinal diseases, which are the topic of this special issue in Visceral Medicine.

Böhm [1] addresses the link between EBW and diverticular disease. The author begins by reviewing prospective studies and meta-analyses performed to date which overall argue that overweight and obesity, particularly central obesity, increase the risk of diverticular disease with a potential pathogenic role for gut microbiota-derived methane. Interestingly, studies are highlighted which argue for sexual dimorphism in diverticulosis development, being mainly found in postmenopausal women possibly from the loss of a protective effect of ovarian steroid hormones. Diverticular bleeding is then discussed which is shown to be increased in both obese men and women, although the underlying triggers of vascular injury and media atrophy remain speculative. Evidence is presented on how EBW promotes the progression from diverticulosis to diverticulitis. It is proposed that the "leaky gut" in obesity could promote inflammation of adjacent mesenteric fat and promote its encroachment onto diverticula, analogous to the "creeping fat" originally described in Crohn's disease. Nevertheless, due caution was advised when trying to associate any particular mucosal microbiota species to disease progression. In terms of predicting diverticulitis, obesity did not have much pow- er in Western populations but did for Eastern populations, with evidence of right-sidedness in South Korean individuals and left-sidedness in Japanese individuals, and which again associated most tightly with central obesity. In contrast, obesity did generally predict recurrent diverticulitis.

Dietz et al. [2] review the link between EBW and abdominal hernia. The authors begin by outlining the multifaceted challenges of dealing with hernias in obese patients, which are twice as common in ventral (umbilical/ epigastric) regions because of the fact that central obesity increases intra-abdominal pressure. The authors then stress the need to use metrics other than BMI to more accurately reflect hernia risk such as abdominal circumference and waist-to-hip-ratio. The authors continue by outlining the prioritization strategy for hernia repair. While in emergency cases the need for immediate surgery is obvious, in elective cases prior weight loss is recommended although minimally invasive mesh implantation in the intraperitoneal position suffices for small hernias in individuals with obesity grades 1 and 2 (BMI $30-40 \mathrm{~kg} / \mathrm{m}^{2}$ ). For those individuals with severe obesity (BMI $>40 \mathrm{~kg} /$ $\mathrm{m}^{2}$ ), the evidence suggests that bariatric surgery prior to hernia repair, rather than during hernia repair, is the best way forward to minimize postoperative morbidity and recurrence rates even for very large ventral hernias. The authors then mention the clear advantages of laparoscopic hernia repair in obese patients but are mindful of the technical challenges that this presents to the surgeon. For these reasons, the authors promote robotic-assisted repair as the ideal approach, especially as it affords free placement of the mesh from a variety of different planes beyond the intraperitoneal one such as retromuscularly. Neverthe- 
less, despite increased usage of robot-assisted hernia repair for obese patients, the authors stress the need for standardized guidelines and recommendations. The authors conclude by summarizing data of the increased risk of groin hernia with increasing BMI, and again how this is best approached using robotic-assisted surgery.

Stokes and Lammert [3] review the link between EBW and gallstone disease. The authors begin by highlighting how both genetic and environmental factors contribute to the development of gallstones. Numerous studies are then presented which clearly show that increased BMI and especially central obesity increase the risk of developing symptomatic gallstones and thus the need for cholecystectomy. Mechanistic insight for the development of cholesterol gallstones in the gallbladder in obesity is then provided: hepatocytes produce more cholesterol as a direct function of increased body fat leading to the accumulation of cholesterol monohydrate crystals in the gallbladder. This, the authors explain, is exacerbated by the reduced gallbladder motility with increased body weight. Further, the authors mention how pro-inflammatory cytokines such as interleukin 6 (IL-6), which are increased in obesity, may independently contribute to the formation of new gallstones although the precise signaling cascades still need to be elucidated. The authors then discuss an interesting and clinically important phenomenon in gallstone disease. While weight loss generally reduces the risk of gallstones, extreme or rapid weight loss, as occurs with bariatric surgeries including sleeve gastrectomy, Roux-en-Y gastric bypass, and biliopancreatic diversion (but not gastric banding) increase the risk of gallstones as does weight cycling. The authors then elaborate on how the complex relationship between bariatric surgery and gallstones extends to cholecystectomy treatment, which is performed during bariatric surgery only for symptomatic gallstone disease. Given these caveats, the authors recommend treating gallstones through changes in diet such as reducing fat and sugar consumption as well as increasing fiber consumption. Additionally, the authors present several studies showing that exercise confers protective effects against the formation of gallstones, but has limited efficacy as an intervention. Finally, when bariatric surgery is necessary, the authors recommend based on the existing literature that $500-600 \mathrm{mg} /$ day of the secondary bile acid ursodeoxycholic acid (UDCA) be administered for 6 months as a prophylaxis against gallstones.

Scherübl [4] reviews the link between EBW and gastrointestinal cancer which encompasses esophageal (adenocarcinoma), gastric (cardia), pancreatic, biliary, hepatocellular, and colorectal cancer. The author then provides a mechanistic basis for cancer development in obesity, which includes insulin resistance, disturbances in the insulin-like growth factor 1 (IGF-1) system and sex hormones, low-grade inflammation and oxidative stress as well as the intestinal microbiota. For example, the author elaborates on how the increased IGF-1 in obesity contributes to colorectal cancer. Pancreatic cancer is then discussed which is predicted to become the second most common cause of cancer-related death in the USA by 2030. The author highlights that pancreatic cancer risk increase by $10 \%$ per $5 \mathrm{~kg} / \mathrm{m}^{2}$ increase in BMI. Cancer risk can be reduced considerably by modifying lifestyle risk factors such as tobacco smoking, alcohol use, and EBW. The author acknowledges that at present primary prevention offers the best chances for reducing pancreatic cancer deaths. As EBW increases the risk of gallstones, which in turn increase the risk of gallbladder cancer, gallstones, and inflammation are the likely link between EBW and gallbladder cancer risk. The author also mentions that cancers of the upper gastrointestinal tract, that is, of the distal esophagus, esophagogastric junction, and gastric cardia are on the rise, which has been attributed to the increased incidence of gastroesophageal reflux disease, diabetes, and obesity. Strikingly, per $5 \mathrm{~kg} / \mathrm{m}^{2}$ increase in BMI the risk of developing esophageal adenocarcinoma increases by approximately $50 \%$ and that of cardia cancer by approximately $25 \%$.

Thalheimer and Bueter [5] review the link between EBW and gastroesophageal reflux disease which shows a prevalence of $30 \%$ in the general population and up to $70 \%$ in patients with severe obesity. This was explained in deep technical detail by the authors to be due to a combination of hiatal hernia, decreased lower esophageal sphincter pressure, and increased intra-abdominal pressure from increased central adiposity. The authors then discuss the treatment options for gastroesophageal reflux disease beyond proton-pump inhibitors and fundal plication with particular emphasis placed on the merits of gastric bypass surgery. They carefully elaborate on how Roux-en-Y gastric bypass achieves its unique beneficial effects not only by inducing weight loss but also by reducing intra-abdominal pressure and directing gastric contents and gastric acid away from the esophagus by a biliopancreatic limb and gastric pouch, respectively. Additionally, the authors mention how Barrett's esophagus, which is twice as common in obesity, is reduced in most patients after Roux-en-Y gastric bypass making it the gold-standard treatment for gastroesophageal reflux disease in the USA. Directly contrasting with Roux-en-Y gastric bypass, the authors add that sleeve gastrectomy tends to aggravate or even de novo cause gastroesophageal reflux disease, esophagitis, and Barrett's esophagus. They explain how this is directly attributable to the new anatomy postoperatively as intragastric pressure is increased while both gastric acid and bile acid reflux is promoted. Because sleeve gastrectomy has recently superseded Roux-en-Y gastric bypass to become the most frequently performed bariatric surgery worldwide, this 
raises legitimate concerns about the long-term consequences on gastroesophageal reflux disease, Barrett's esophagus, and esophageal cancer incidence in the future.

Roeb [6] reviews the link between EBW and nonalcoholic fatty liver disease which afflicts about $30 \%$ of the world's population. The author cautions that the newly coined term metabolic dysfunction associated fatty liver disease which is diagnosed based on the presence of overweight/obesity, diabetes, and cardiovascular problems might overlook the significant number of individuals with NAFLD who do not have these conditions. As an example, the author refers to the Tübingen study that found that nonobese patients with prediabetes had a several-fold increased risk of NAFLD. The author discusses the complex etiology of NAFLD which includes increased hepatic lipogenesis from increased glucose, fructose, and saturated fatty acid consumption, inflammation, and intestinal barrier dysfunction. The author adds that mitochondrial dysfunction and oxidative stress also occur in hepatocytes during NAFLD pathogenesis and that hyperinsulinemia increases hepatic lipogenesis as well as dysregulated lipolysis of white adipose tissue. The author concludes that lifestyle modifications such as increasing physical activity and protein intake as well as weight loss are recommended. The author also points out that bariatric surgery is a very effective treatment for NAFLD and that several drugs are in the pipeline such as the nuclear bile acid receptor FXR agonist obeticholic acid.

Wiese et al. [7] review the link between EBW and pancreatic disease which the authors' reason is a continuum from acute pancreatitis, through to recurrent acute pancreatitis, chronic pancreatitis, and then to pancreatic cancer. The authors argue how definitions of obesity may confound an association with pancreatic disease and how an obesity paradox complicates matters further. As concerns acute pancreatitis, in which there are a decent number of studies published, waist circumference and central obesity consistently increase risk. For chronic pancreatitis, however, fewer studies exist and these suggest an inverse effect of increased BMI most likely due to malnutrition, maldigestion, and weight loss usually associated with chronic pancreatitis. EBW is a well-known risk factor of pancreatic cancer. The authors discuss some mech- anisms of how EBW might cause pancreatic disease. Intrapancreatic fat as well as immune dysfunction is main suspects. To explain the paradoxical "protective" effect of increased body weight in chronic pancreatitis, the authors refer to fibrotic tissue surrounding intrapancreatic adipocytes which shield from lipolysis. In pancreatic cancer however, the author says that in obesity the increased IGF1 and insulin levels might stimulate cell proliferation and invasion.

Finally, Plaßmeier et al. [8] review the impact of EBW on postsurgical complications. They find that while obesity itself is not a consistent predictor of postsurgical complications, comorbidities such as diabetes, chronic heart failure, or kidney failure are. The authors conclude that the technical challenges of operating on obese patients increase surgery times, which may increase postsurgical complications.

The multidisciplinary discussion is chaired by Kähler et al. [9]. The discussion sheds light on the challenges posed by the timely work-up of obese patients presenting with acute abdominal pain and/or signs of infection. The authors advise for special attention in the care of patients with EBW.

The articles of this special issue of Visceral Medicine provide readers with an up-to-date overview of the impact of EBW on various gastrointestinal diseases. We hope that the outlined developments will inspire new diagnostic and therapeutic strategies and will optimize the care of patients suffering from EBW.

\section{Conflict of Interest Statement}

The authors have no conflicts of interest to declare.

\section{Funding Sources}

There was no funding for this editorial.

\section{Author Contributions}

Germer C. and Scherübl H. jointly planned, wrote, and revised the editorial.

\section{References}

1 Böhm S. EBW and diverticular disease. Visc Med. 2021. doi: 10.1159/000518674.

2 Dietz U, Kudsi OY, Gokcal F, Bou-Ayash N, Pfefferkorn U, Rudofsky G, et al. EBW and abdominal hernias. Visc Med. 2021. doi: 10.1159/000516047 [Epub ahead of print].

3 Stokes CS, Lammert F. EBW and gallstone disease. Visc Med. 2021. doi: 10.1159/000516418 [Epub ahead of print].
4 Scherübl H. EBW and gastrointestinal cancer risk. Visc Med. 2021. doi: 10.1159/000515444 [Epub ahead of print].

5 Thalheimer A, Bueter M. EBW and gastroesophageal reflux disease. Visc Med. 2021. doi: $10.1159 / 000516050$ [Epub ahead of print].

6 Roeb E. EBW and metabolic associated fatty liver disease (MAFLD). Visc Med. 2021. doi: $10.1159 / 000515445$ [Epub ahead of print].
7 Wiese ML, Aghdassi AA, Lerch MM, Steveling A. EBW and pancreatic disease. Visc Med. 2021. doi: 10.1159/000517147 [Epub ahead of print].

8 Plaßmeier L, Hankir MK, Seyfried F. Impact of EBW and post-surgical complications. Visc Med. 2021. doi: 10.1159/000517345 [Epub ahead of print]

9 Kähler G, Otto M, Kahl S, Willeke F, Sendt W, Gossner L. Interdisciplinary discussion. Visc Med. 2021. doi: 10.1159/000516785 [Epub ahead of print]. 